\title{
Improved arterial stiffness in mitral stenosis after successful percutaneous balloon valvuloplasty
}

\author{
Ibrahim Ozdogru ${ }^{1}$, Ahmet Celik ${ }^{2}$, Ali Dogan ${ }^{1}$, Ozcan Orscelik $^{1}$, Omer Sahin ${ }^{1}$, \\ Deniz Elcik ${ }^{1}$, Tolga Saka ${ }^{3}$, Ramazan Topsakal ${ }^{1}$, Abdurrahman Oguzhan ${ }^{1}$ \\ ${ }^{1}$ Department of Cardiology, Erciyes University Medical Faculty, Kayseri, Turkey \\ ${ }^{2}$ Department of Cardiology, Elazig Education and Research Hospital, Elazig, Turkey \\ ${ }^{3}$ Department of Sports Medicine, Erciyes University Medical Faculty, Kayseri, Turkey
}

\begin{abstract}
Background: Rheumatic mitral stenosis (MS) is still a common disease in developing countries with high morbidity and mortality rates. The purpose of the study was to evaluate arterial stiffness in severe MS before and after percutaneous mitral balloon valvuloplasty $(P M B V)$.
\end{abstract}

Methods: Thirty patients with MS in sinus rhythm requiring PMBV and 20 age-gender matched healthy volunteers. The analyze of pulse wave velocities $(P W V)$ were performed using of the carotid artery at the femoral by PWV technique on patients at baseline and a week after $P M B V$.

Results: The values of PWV were significantly decreased after successful PMBW in $M S$ patients. Mitral mean gradients and systolic pulmonary artery pressures (sPAP) both on echocardiography and catheterization also had a significant decrease after PMBW. The mitral valve areas were significantly increased after $P M B W$. There was a highly significant negative correlation between mitral valve areas and PWV values. A highly significant positive correlation was seen between mitral mean gradient on catheterization and $P W V(r=0.830$, $p<0.001)$. There was also a significant correlation between sPAP on catheterization and $P W V$ values $(r=0.639, p<0.001)$. Echocardiographic mitral mean gradients and $P W V$ were highly positive correlated with each other $(r=0.841, p<0.001)$. The sPAP on echocardiography had also a highly positive correlation with $P W V(r=0.681, p<0.001)$.

Conclusions: Mitral stenosis is a cause of impaired arterial stiffness and after the enlargened mitral valve area arterial stiffness improved in patients with MS. (Cardiol J 2012; 19, 6: 586-590)

Key words: arterial stiffness, mitral stenosis, valvuloplasty, pulse wave velocity

\section{Introduction}

Several studies had shown an association between increased arterial stiffness and aging [1], diabetes mellitus [2], hypercholesterolemia [3], hypertension [4], smoking [5], congestive heart fail- ure [6] and chronic kidney disease [7]. Pulse wave velocity (PWV) is used to measure arterial elasticity and stiffness and is related to the elastic properties of the vascular wall. Carotid-femoral PWV is considered the gold-standard measurement of central arterial stiffness [8].

Address for correspondence: Ahmet Celik, MD, Elazig Education and Research Hospital, Department of Cardiology, Elazig, Turkey, tel: +90 53179279 10, fax: +90 42421214 61, e-mail: ahmetcelik39@hotmail.com

Received: 03.07.2012 Accepted: 01.08.2012 
Rheumatic mitral stenosis (MS) is still a common disease in developing countries with high morbidity and mortality rates [9]. The safe and effective treatment of choice for MS patients with favorable mitral valve morphology is percutaneous balloon mitral valvuloplasty (PMBV). Successful PMBV cause significant improvements in early clinical and hemodynamic properties of patients with MS. While mitral valve area (MVA) increases, the left atrial pressure, mean transmitral gradient and pulmonary artery pressure decrease immediately.

To the best of our knowledge there was no data about the relationship between MS and arterial stiffness in the literature. In this study, we aimed to show not only the effect of MS on arterial stiffness but also the change of arterial stiffness after successful PMBV.

\section{Methods}

We prospectively screened 30 consecutive patients with rheumatic MS in sinus rhythm (MS Group) whom required PMBV and 20 age and sexmatched healthy volunteers (Healthy Group). Patients with moderate or severe mitral regurgitation, any moderate or severe valvular heart disease except MS, history of hypertension, coronary artery disease, hypercholesterolemia, diabetes mellitus, chronic kidney disease, current smoking, body mass index higher than 25 were exluded from the study. All patients and volunteers were informed about the study, and their written consent forms were obtained. The study was approved by the local ethic committee, performed in accordance to the Declaration of Helsinki.

\section{Pulse wave velocity}

Vascular studies were performed in a quiet, temperature controlled room with subjects resting in a supine position at baseline and a week after PMBV. Systolic and diastolic blood pressures were measured twice using a semi-automated non-invasive oscillometric sphygmomanometer, following a 10 min rest period on the dominant arm of subjects. Pulse wave analysis was performed using the carotid artery at the femoral by PWV machine (Micro medical Pulse Trace, Rochester, UK) in accordance with the manufacturer's recommendations before treadmill exercise testing. PWV was calculated by measuring the time for the pulse wave to travel between the carotid and femoral arteries. All measurements were performed by a single operator blinded to the nature of each exposure.
PWV was determined by means of a noninvasive analysis of the propagation time and distance of the pulse wave between two acquisition points: $\mathrm{PWV}=$ distance $[\mathrm{m}] /$ time $[\mathrm{s}]$. The transducers were positioned over the carotid and femoral arteries, always on the right side of the body and the signals were sent to the Complior system (France). Signal acquisition was performed by the same researcher who was blinded to the patient's condition position before and after exertion on a stationary bike, with 15 sequential pulse waves preferentially recorded. The individual returned to the evaluation bed for the acquisition of the data as soon as the predicted heart rate was reached or other exercise interruption criteria were fulfilled.

\section{Echocardiography}

The echocardiographies were carried out by a cardiology specialist in the echocardiography laboratory in our cardiology department at baseline and a week after PMBV. The echocardiography was performed by Vivid 7 instruments (GE Medical Systems, Milwaukee, WI, USA), with a 2.5-MHz transducer and harmonic imaging. According to the recommendations of the American Society of Echocardiography [10], all echocardiographic examinations were performed with the patient lying in the left lateral decubitus position, and 2-dimensional images were recorded and measured at the apical 4-chambers, 2-chambers, parasternal long- and short-axis views. Mitral valve area was calculated by planimetric method. Diastolic transmitral gradients were measured by continuous-wave Doppler echocardiography. Systolic pulmonary artery pressure (sPAP) was measured with continuous wave Doppler. Tricuspid regurgitation velocity (V) recorded from any view and used to determine sPAP $\left(\mathrm{sPAP}=4 \mathrm{~V}^{2}+\right.$ right atrial pressure $)$. Right atrial pressure was calculated using the caval respiratory index as described by Kircher et al. [11].

\section{Catheterization}

Cardiac catheterization was performed with Philips Integris 5000 equipment (Philips Medical Systems, Best, Netherlands). sPAPs and mitral gradients were also measured with cardiac catheterization. PMBVs were performed using the Inoue balloon technique.

\section{Statistical analysis}

Categorical variables were presented as frequencies and percentages and were compared with the $\chi^{2}$ test. Continuous variables were expressed 
Table 1. Clinical characteristics of study patients.

\begin{tabular}{lccc}
\hline Variables & Mitral stenosis group $(\mathbf{n}=\mathbf{3 0})$ & Healthy group $(\mathbf{n}=\mathbf{2 0})$ & $\mathbf{P}$ \\
\hline Age [years] & $40 \pm 9$ & $40 \pm 6$ & 0.9 \\
Female [\%] & 53 & 60 & 0.4 \\
Creatinine [mg/dL] & $0.95 \pm 0.16$ & $0.92 \pm 0.14$ & 0.5 \\
NYHA functional class & $2.1 \pm 0.5$ & - & 0.9 \\
Systolic blood pressure [mm Hg] & $120 \pm 14$ & $121 \pm 11$ & 0.3 \\
Diastolic blood pressure [mm Hg] & $70 \pm 9$ & $67 \pm 7$ & $72 \pm 07$ \\
Heart rate [bpm] & $89 \pm 11$ & 20.001 \\
\hline
\end{tabular}

Data expressed as mean $\pm \mathrm{SD}$ or percentage. $\mathrm{P}<0.05$ was accepted as a statistically significant. Variables were recorded before the procedure.

Table 2. Echocardiographic, catheterization and pulse wave velocities data for all study patients before and after percutaneous mitral balloon valvuloplasty (PMBV).

\begin{tabular}{lccc}
\hline & Before PMBV & After PMBV & P \\
\hline Pulse wave velocity [m/s] & $10.5 \pm 1.6$ & $5.9 \pm 10$ & $<0.001$ \\
Echocardiographic data: & & & $<0.001$ \\
$\quad$ Mitral valve area $\left[\mathrm{cm}^{2}\right]$ & $1.0 \pm 0.1$ & $2.0 \pm 0.1$ & $<0.001$ \\
Mean gradient $[\mathrm{mm} \mathrm{Hg}]$ & $15 \pm 3$ & $4 \pm 0.9$ & $<0.001$ \\
$\quad$ Systolic PAP $[\mathrm{mm} \mathrm{Hg}]$ & $57 \pm 15$ & $35 \pm 8$ & $<0.001$ \\
Catheterization data: & $18 \pm 4$ & $4 \pm 1$ & $<0.001$ \\
$\quad$ Mean gradient $[\mathrm{mm} \mathrm{Hg}]$ & $54 \pm 18$ & $33 \pm 10$ & \\
Systolic PAP $[\mathrm{mm} \mathrm{Hg}]$ & & & \\
\hline
\end{tabular}

Data expressed as mean $\pm \mathrm{SD} . \mathrm{P}<0.05$ was accepted as a statistically significant; PAP — pulmonary artery pressure

as means and SD. A value of $\mathrm{p}<0.05$ was considered to be significant. To compare the measurements before and after PMBV, student paired t test was used. Correlation analyses were performed using the Pearson coefficient of correlation. The SPSS 15.0 software was used for basic statistical analysis (Version 15, SPSS Inc., Chicago, IL, USA).

\section{Results}

The baseline clinical and demographic properties of all study subjects were seen in Table 1 . There were no significant differences between the MS group and Healthy Group with respect to age, sex, serum creatinine, systolic and diastolic blood pressure ( $p>0.05)$. The baseline heart rate of MS patients were higher than healthy volunteers (Table 1). All patients with MS had successful PMBV without any complication.

No significant differences was found between heart rate at PWV measure before PMBV and after PMBV (70 \pm 6 and $69 \pm 5, \mathrm{p}=0.4$, respectively).

The values of PWV were significantly decreased after successful PMBW. Both mitral mean gradient and sPAP on echocardiography and catheterization also had a significant decrease after successful PMBW. The MVA that measured with plannimetric method on echocardiography had a significant increase after successful PMBW (Table 2).

There was a highly significant negative correlation between MVA and PWV values (Fig. 1). A highly significant positive correlation was seen between mitral mean gradient on catheterization and PWV $(\mathrm{r}=0.830, \mathrm{p}<0.001)$. There was also a significant correlation between sPAP on catheterization and PWV values $(r=0.639, p<0.001)$.

Echocardiographic mitral mean gradient and PWV was highly positive correlated with each other $(r=0.841, p<0.001)$. The sPAP on echocardiography had also a highly positive correlation with PWV ( $\mathrm{r}=0.681, \mathrm{p}<0.001)$.

\section{Discussion}

This study demonstrated that arterial stiffness as measured by PWV improves after successful PMBV. Furthermore, the greater the improvement in MVA then the greater the improvement in arte- 


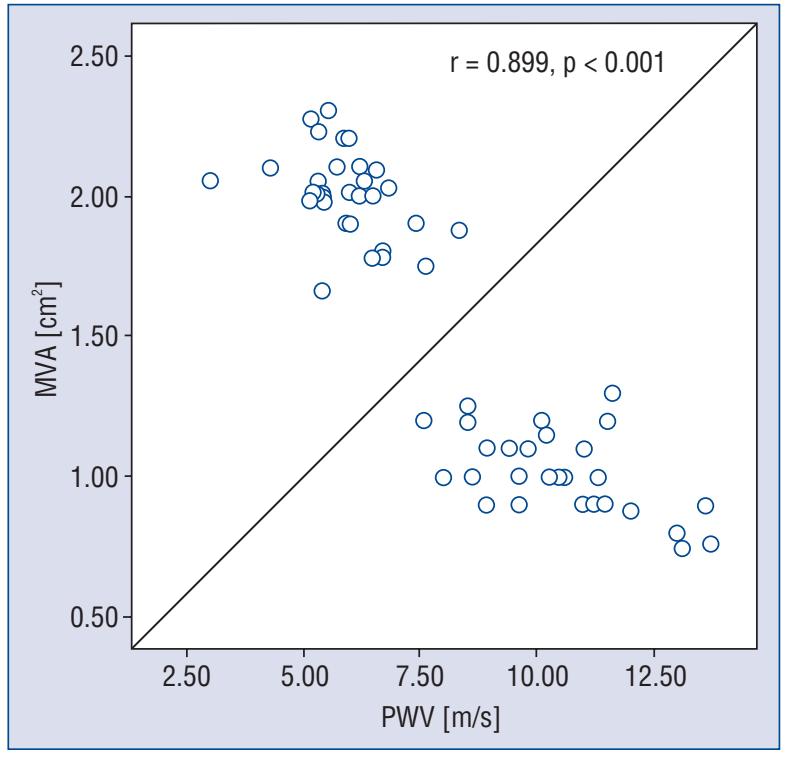

Figure 1. The correlation analysis of mitral valve area (MVA) and pulse wave velocity (PWV) values.

rial stiffness (as measured by PWV). This is the first study demonstrating the worsened arterial stiffness by high PVW levels in MS and the improved arterial stiffness after mitral balloon valvuloplasty. The highly significant positive correlation between PWV and MVA were also firstly showed in this study. We found that different degrees of MVA worsened arterial stiffness measured by PWV according to severity of MVA. With our findings it can be said that the decrease of MVA have directly negative effect on arterial stiffness.

MS is an obstruction to left ventricular inflow at the level of the mitral valve as a result of a structural abnormality of the mitral valve apparatus, which prevents proper opening during diastolic filling of the left ventricle [12]. The major cause of MS is rheumatic fever [13]. Rheumatic MS occurs as a late sequela of rheumatic fever and is still an important condition in developing countries. PMBV is a preferred treatment for symptomatic patients and favorable anatomical findings with moderate or severe MS without left atrial thrombus and significant mitral regurgitation [12]. It has many beneficial effects at early and late period after successful balloon valvuloplasty.

The increase in left atrial pressure and transmitral flow and the decrease in the diastolic filling period are major causes of symptoms in MS. The low cardiac output and increased pulmonary arteriolar resistance result functional and structural changes such as alveolar basement membrane thickening, adaptation of neuroreceptors, increased lymphatic drainage, and increased transpulmonary endothelin spillover rate in patients with severe MS [14-16]. MS is a progressive and lifelong disease. We hypothesize that it may also cause worsening in stiffness of peripheral arteries. PMBV, which is the treatment of choice in patients with MS with favorable mitral valve morphology, is unique in that it provides dramatic hemodynamic and symptomatic relief as soon as it is carried out with success. Due to these sudden changes in hemodynamic and metabolic parameters in MS after PMBV, recently, it seemed to be highly attractive to investigate some characteristics of MS before and after the procedure by many clinicians.

Arterial stiffness is an important predictor to the development of cardiovascular disease. There are studies which showed acute change of arterial stiffness in some conditions [17-21]. It was shown that heart failure patients with normal ejection fraction had higher PWV values than healthy subjects and reduced aortic compliance might be a major risk factor for heart failure with normal ejection fraction [22]. Another study showed lower PWV values were related with less short-term cardiovascular events in acute heart failure patients [23]. Radaelli et al. [24] demonstrated the correlation between imparied baroreflex sensitivity and increased PWV values in chronic heart failure and coronary artery disease patients. It was known that patients with MS had increased sempatic activity and impaired baroreflex sensitivity [25]. In some studies, it was found that PMBV improves the baroreflex sensitivity and sempatic activity in MS patients $[25,26]$. In light of this data we thought that with respect to patients with MS have impaired baroreflex sensitivity and sempatic activity, so they might have reduced aortic compliance. In our study, we have found higher PWV values in MS patients than healthy controls and showed increased arterial stiffness in MS and improvement of arterial stiffness after successful treatment of MS with PMBV. The MS should be treated not only avoid pulmonary edema but also the negative effect of increased arterial stiffness which is a strong predictor of future cardiovascular events and all-cause mortality [27].

\section{Conclusions}

This study demonstrated that MS is a cause of worsened arterial stiffness and after the enlargened MVA, arterial stiffness improved in patients with MS.

Conflict of interest: none declared 


\section{References}

1. Mitchell GF, Parise H, Benjamin EJ et al. Changes in arterial stiffness and wave reflection with advancing age in healthy men and women: The Framingham Heart Study. Hypertension, 2004; 43: 1239-1245.

2. Tedesco MA, Natale F, Salvo GD, Caputo S, Capasso M, Calabro R. Effects of coexisting hypertension and type II diabetes mellitus on arterial stiffness. J Hum Hypertens, 2004; 18: 469-473.

3. Sharman JE, McEniery CM, Dhakam ZR, Coombes JS, Wilkinson IB, Cockcroft JR. Pulse pressure amplification during exercise is significantly reduced with age and hypercholesterolemia. J Hypertens, 2007; 25: 1249-1254.

4. Wallace SM, Yasmin, McEniery CM et al. Isolated systolic hypertension is characterized by increased aortic stiffness and endothelial dysfunction. Hypertension, 2007; 50: 228-233.

5. Mahmud A, Feely J. Effect of smoking on arterial stiffness and pulse pressure amplification. Hypertension, 2003, 41: 183-187.

6. Mitchell GF, Tardif JC, Arnold JM et al. Pulsatile hemodynamics in congestive heart failure. Hypertension, 2001; 38: 1433-1439.

7. Matsuda N, Takei T, Fujiu A, Ogawa T, Nitta K. Arterial stiffness in patients with non-diabetic chronic kidney disease (CKD). J Atheroscler Thromb, 2009; 16: 57-62.

8. O'Rourke MF, Franklin SS. Arterial stiffness: Reflections on the arterial pulse. Eur Heart J, 2006; 27: 2497-2498.

9. Bonow RO, Carabello BA, Chatterjee K et al. 2008 Focused update incorporated into the ACC/AHA 2006 guidelines for the management of patients with valvular heart disease: A report of the American College of Cardiology/American Heart Association Task Force on Practice Guidelines (Writing Committee to Revise the 1998 Guidelines for the Management of Patients With Valvular Heart Disease): Endorsed by the Society of Cardiovascular Anesthesiologists, Society for Cardiovascular Angiography and Interventions, and Society of Thoracic Surgeons. Circulation, 2008; 118: 523-661.

10. Lang RM, Bierig M, Devereux RB et al. Recommendations for chamber quantification: A report from the American Society of Echocardiography's Guidelines and Standards Committee and the Chamber Quantification Writing Group, developed in conjunction with the European Association of Echocardiography, a branch of the Europea Society of Cardiology. J Am Soc Echocardiography, 2005; 18: 1440-1463.

11. Kircher BJ, Himelman RB, Schiller NB. Noninvasive assessment of right atrial pressure from inspiratory collapse of the inferior vena cava. Am J Cardiol, 1990; 66: 493-496.

12. Bonow RO, Carabello BA, Chatterjee K et al.; American College of Cardiology/American Heart Association Task Force on Practice Guidelines. 2008 focused update incorporated into the ACC/ /AHA 2006 guidelines for the management of patients with valvular heart disease: a report of the American College of Cardiology/American Heart Association Task Force on Practice Guidelines (Writing Committee to revise the 1998 guidelines for the management of patients with valvular heart disease). Endorsed by the Society of Cardiovascular Anesthesiologists, Society for Cardiovascular Angiography and Interventions, and Society of Thoracic Surgeons. J Am Coll Cardiol, 2008; 52: e1-e142.

13. Bonow RO, Braunwald E. Valvular heart disease. In: Braunwald E ed. Heart disease. $7^{\text {th }}$ Ed. Saunders Company, Philadelphia 2005: 1553-1632.

14. Hugenholtz PG, Ryan TJ, Stein SW, Belmann WH. The spectrum of pure mitral stenosis: hemodynamic studies in relation to clinical disability. Am J Cardiol, 1962; 10: 773-784.

15. Gorlin R. The mechanism of the signs and symptoms of mitral valve disease. Br Heart J, 1954; 16: 375-380.

16. Wood P. An appreciation of mitral stenosis, II: Investigations and results. Br Med J, 1954; 4871: 1113-1124.

17. Tordi N, Mourot L, Colin E, Regnard J. Intermittent versus constant aerobic exercise: effects on arterial stiffness. Eur J Appl Physiol, 2010; 108: 801-809.

18. Heffernan KS, Rossow L, Jae SY, Shokunbi HG, Gibson EM, Fernhall B. Effect of single-leg resistance exercise on regional arterial stiffness. Eur J Appl Physiol, 2006; 98: 185-190.

19. Heffernan KS, Collier SR, Kelly EE, Jae SY, Fernhall B. Arterial stiffness and baroreflex sensitivity following bouts of aerobic and resistance exercise. Int J Sports Med, 2007; 28: 197-203.

20. LeBeouf A, Mac-Way F, Utescu MS et al. Effects of acute variation of dialysate calcium concentrations on arterial stiffness and aortic pressure waveform. Nephrol Dial Transplant, 2009; 24: 3788-3794.

21. Dhaun N, Macintyre IM, Melville V et al. Blood pressure-independent reduction in proteinuria and arterial stiffness after acute endothelin-a receptor antagonism in chronic kidney disease. Hypertension, 2009; 54: 113-119.

22. Ibrahim el-SH, Miller AB, White RD. The relationship between aortic stiffness and $\mathrm{E} / \mathrm{A}$ filling ratio and myocardial strain in the context of left ventricular diastolic dysfunction in heart failure with normal ejection fraction: insights from magnetic resonance imaging. Magn Reson Imaging, 2011; 29: 1222-1234.

23. Sung SH, Yu WC, Cheng HM et al. Pulsatile hemodynamics and clinical outcomes in acute heart failure. Am J Hypertens, 2011; 24: 775-782.

24. Radaelli A, Castiglioni P, Balestri G et al. Increased pulse wave velocity and not reduced ejection fraction is associated with impaired baroreflex control of heart rate in congestive heart failure. J Hypertens, 2010; 28: 1908-1912.

25. Ashino K, Gotoh E, Sumita S, Moriya A, Ishii M. Percutaneous transluminal mitral valvuloplasty normalizes baroreflex sensitivity and sympathetic activity in patients with mitral stenosis. Circulation, 1997; 96: 3443-3449.

26. Yuasa $\mathrm{T}$, Takata $\mathrm{S}$, Terasaki $\mathrm{T}$ et al. Percutaneous transluminal mitral valvuloplasty improves cardiopulmonary baroreflex sensitivity in patients with mitral stenosis. Auton Neurosci, 2001; 94: 117-124.

27. Vlachopoulos C, Aznaouridis K, Stefanadis C. Prediction of cardiovascular events and all-cause mortality with arterial stiffness: A systematic review and meta-analysis. J Am Coll Cardiol, 2010; 55: 1318-1327. 